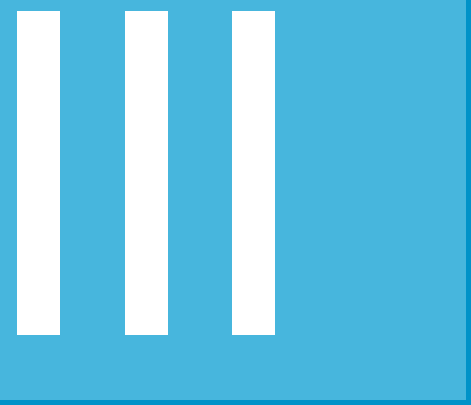

\title{
ESTUDIO DE LA COMPOSICIÓN CORPORAL Y LA APTITUD FÍSICA EN ESTUDIANTES DEL PRIMER CICLO DE LA UNIVERSIDAD PERUANA UNIÓN - TARAPOTO 2012
}

Research of the corporal composition and the physical aptitude in students from first cycle of Union Peruvian University, Tarapoto, 2012

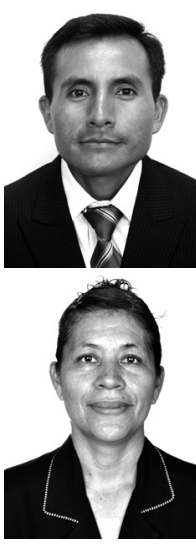

\section{Saulo Salinas}

Licenciado en Educación Física por la Universidad Nacional Enrique Guzmán y Valle. Magíster en Ciencias del Deporte por la Universidad Nacional Enrique Guzmán y Valle. Profesor universitario y coordinador de Estilo de Vida y Deportes de la Universidad Peruana Unión. En la actualidad se desempeña como director de Extensión y Proyección Social de la Universidad Peruana Unión, sede Tarapoto.

\section{Norma Alejandría Lozano}

Licenciada en Educación en la especialidad de Educación Física por la Universidad Pedro Ruiz Gallo, Lambayeque. Magíster en Ciencias de la Educación con mención en Investigación y Docencia. Profesora del Instituto Superior Pedagógico Público Tarapoto. Profesora en Cultura Física de la Universidad Peruana Unión, sede Tarapoto. 


\section{Resumen}

En años pasados, el cáncer llegó a ser la enfermedad mortal número uno a escala mundial y hoy es la obesidad, que se presenta sin hacer distinción de edad, sexo, nivel socioeconómico ni religión. El objetivo del estudio fue describir la composición corporal y la aptitud física asociada a la salud en los estudiantes de la Universidad Peruana Unión - Tarapoto. La muestra de estudio fue conformada por 318 estudiantes, de ambos sexos, en edades de 16 a 19 años. Tuvo un diseño no experimental de tipo descriptivo. En la composición corporal se obtuvo el índice de masa corporal (IMC) y el perfil de adiposidad. Para describir la aptitud física asociada a la salud se tomó cinco pruebas: carrera de la milla, push up, trunk lift, abdominales 30', y burpee 30'. Los resultados muestran que al evaluar el IMC en los estudiantes, se obtuvo un $54.72 \%$ por debajo del peso ideal, $26.73 \%$ en el peso ideal y un $18.55 \%$ se encuentra entre la preobesidad y la extrema obesidad. Los valores medios de los varones entre 16 y 19 años en la suma de tres pliegues fueron de $38.8 \%$ y $36.9 \%$, a diferencia de los valores medios presentados por el sexo femenino; sin embargo, los varones entre 17 y 18 años presentan el $38.5 \%$ y $36.6 \%$ lo que indica que incrementaron sus valores medios en contraste a las mujeres. Asimismo, en las pruebas de la aptitud física, en ambos sexos, existe un incremento significativo de los valores medios a favor de los varones, excepto en la prueba de la milla donde, a los 19 años, las mujeres obtienen un menor desempeño.

Palabras clave: Composición corporal, aptitud física y universitarios.

\section{Abstract}

In the last ages cancer became the mortal number one illness in global scale, nowadays is the obesity, which presents without any distinction of age, gender, socioeconomic level neither religion. The target of the study was to describe the corporal composition and the physical aptitude associated to health of students of Union Peruvian College - Tarapoto. The sample of the study was conformed of 318 students, both genders, between the ages from 16 to 19 . The research design is no experimental descriptive. In corporal composition we obtained the (BMI) body mass index and to describe the physical aptitude associated to health we take five tests: mile race, push up, trunk lift, 30 seconds abdominal, and 30 seconds Burpee. The results show that when we evaluate the BMI in the students, we obtained a $54.72 \%$ underneath the right weight, $26.73 \%$ in the right weight and an $18.55 \%$ finds between pre obese and extremely obese. The average values in males of 16 and 19 in the sum of three folds were $38.8 \%$ and $36.9 \%$, unlike the average values displayed by female gender; however, males between 17 and 18 show the $38.5 \%$ and $36.6 \%$ indicating that they increased their average values in contrast to females. Also, in the physical aptitude test, in both genders, there is a significant increase of the average values in favor of males, except in the mile race test when, at the age of 19 , females get lower performance.

Keyword: Corporal composition, physical aptitude and college students 


\section{Introducción}

Los efectos del desarrollo tecnológico, producidos en las últimas décadas, han producido un cambio radical en el estilo de vida de los adolescentes y jóvenes; siendo así que la falta de actividad física forma cada vez más personas sedentarias (Buchner y Milles, 2002; citado por Glaner, 2003). Los efectos de este desarrollo serán las manifestaciones claras de problemas de salud como las enfermedades cardiovasculares, diabetes mellitus tipo 2, obesidad y osteoporosis que se desencadenarán en la adultez (Malina y Strong, 2005; citado por Bustamante, 2007). Frente a una realidad problemática, las universidades, con una responsabilidad importante en la salud, están interesadas en la prevención de enfermedades, como también en destacar la necesidad de lograr que se asuma e interiorice un estilo de vida activo y saludable, que debe ser mantenido a lo largo de la vida.

Como ya lo mencionaban Berman y Braga (2005), niños y adolescentes realizan cada vez menos actividad física, es por eso que se sitúan dentro de los niveles que generan preocupaciones en la comunidad científica en el área de salud y una de las medidas que se toma es considerar a las escuelas y universidades como lugares para la prevención y promoción de la salud.

Si queremos reducir los riesgos de mortandad prematura y de enfermedades crónico-degenerativas y además reducir la obesidad, que es una enfermedad a escala mundial, entonces tenemos que prevenir los riesgos y mejorar nuestro estilo de vida.

Una preocupación creciente, en el curso de Cultura Física, es mantener el equilibrio en el área física, con el número de horas suficientes para lograr el hábito de la actividad física.

Consideramos el interés de describir las características de la composición corporal y aptitud física, asociada a la salud de los estudiantes del $1 .^{\mathrm{er}}$ ciclo de estudios de la Universidad Peruana Unión, Tarapoto.

\section{Objetivo general}

Describir la composición corporal y la aptitud física asociadas a la salud en los estudiantes de ambos sexos del 1 . $^{\text {er }}$ ciclo de estudios de la Universidad Peruana Unión - Tarapoto, 2012. 


\section{Objetivos específicos}

1. Describir las características de la composición corporal en los estudiantes de ambos sexos del 1.er ciclo de la Universidad Peruana Unión - Tarapoto, 2012.

2. Identificar las características de la aptitud física asociadas a la salud en los estudiantes de ambos sexos del 1. ${ }^{\text {er }}$ ciclo de la Universidad Peruana Unión Tarapoto, 2012.

\section{Importancia}

Se justifica por la importancia inequívoca del estudio integrado de la aptitud física y la composición corporal de adolescentes y jóvenes cuyo análisis servirá para investigaciones futuras.

La escasez de informaciones respecto de las variables estudiadas en adolescentes y jóvenes, en la realidad peruana, se constituye en un factor importante de incorporación de nuevos conocimientos de gran relevancia.

\section{Composición corporal}

La composición corporal se refiere a la cantidad y proporción de los diversos componentes químicos del cuerpo humano, los cuales tienen una relación con la salud, las enfermedades y la calidad de vida de cada individuo (Hawes, Eston, Reilly y Stolarczyk, 1996; citado por Bustamante, 2003).

\section{Valoración de la composición corporal}

Para la valoración de la composición corporal se utilizó los métodos doblemente indirectos, donde se obtiene el índice de masa corporal (IMC) para evaluar la normalidad, sobrepeso u obesidad de los estudiantes; asimismo, los pliegues cutáneos del tríceps, subescapular, pantorrilla y la sumatoria de los tres pliegues, para estimar la distribución regional de grasa subcutánea en el tronco y en las extremidades superiores e inferiores (Heyward y Stolarczyk, 2000).

\section{Aptitud física asociada a la salud}

Capacidad de las personas para realizar esfuerzos físicos que posean garantías a su sobrevivencia en buenas condiciones orgánicas en el ambiente en que viven (Bouchard, 1994; citado por Glaner, 2003). 


\section{Población muestra}

El trabajo de investigación se realizó en las instalaciones de la Universidad Peruana Unión, sede Tarapoto.

La población de estudio estaba conformada por 318 estudiantes (172 féminas - 146 varones) del $1 .^{\text {er }}$ ciclo de estudios entre las edades de 16 y 19 años.

Tabla 1.- Estudiantes del 1 er $^{\text {er }}$ ciclo de estudios de la UPeU que fueron evaluados.

\begin{tabular}{cccc}
\hline EDAD & FEMENINO & MASCULINO & TOTAL \\
\hline 16 & 50 & 31 & 81 \\
17 & 56 & 62 & 118 \\
18 & 48 & 36 & 84 \\
19 & 18 & 17 & 35 \\
\hline Total & $\mathbf{1 7 2}$ & $\mathbf{1 4 6}$ & $\mathbf{3 1 8}$ \\
\hline
\end{tabular}

Tipo de estudio y diseño de investigación

El estudio fue descriptivo. Bajo los parámetros de este tipo de estudio se asumió los procedimientos para la adopción del diseño de investigación. Se recolectó información en varias muestras, con respecto a un mismo fenómeno.

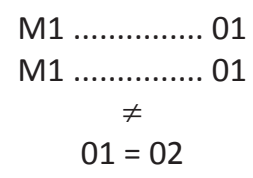

Donde:

$\mathrm{M} 1, \mathrm{M} 2=\mathrm{c} / \mathrm{u}$ de las muestras

01, 02 = Información de cada muestra 


\section{Medición de las variables}

\section{Composición corporal}

En la composición corporal, fueron determinados el índice de masa corporal (peso expresado en kg entre el cuadrado de la altura expresado en metros) y la adiposidad subcutánea fue establecida a partir de la suma de los tres pliegues cutáneos del tríceps, subescapular y pantorrilla.

En el cuadro A se presenta la operacionalización de la variable de la composición corporal.

\section{Cuadro A}

\begin{tabular}{|c|c|c|c|}
\hline VARIABLE & DIMENSIONES & INDICADOR & INSTRUMENTO \\
\hline $\begin{array}{l}\text { COMPOSICIÓN } \\
\text { CORPORAL }\end{array}$ & $\begin{array}{l}\text { - ÍNDICE DE MASA } \\
\text { CORPORAL (IMC) } \\
\text { - PERFIL DE ADI- } \\
\text { POSIDAD }\end{array}$ & $\begin{array}{l}\text { - Altura } \\
\text { - Peso } \\
\text { - Tríceps } \\
\text { - Subescapular } \\
\text { - Pantorrilla Medial }\end{array}$ & $\begin{array}{l}\text { - Estadiómetro } \\
\text { - Báscula } \\
\text { - Calibrador de pliegues } \\
\text { dérmicos }\end{array}$ \\
\hline
\end{tabular}

\section{Aptitud física asociada a la salud}

Para esta variable, hemos utilizado como referencia algunas pruebas de la batería Fitnessgram: carrera de la milla, push up y trunk Lift. Asimismo, hemos agregado 2 pruebas físicas abdominales de 30 segundos, y burpee de 30 segundos. En el cuadro B, se presenta la operacionalización de la variable aptitud física.

\section{Cuadro B}

\begin{tabular}{|c|c|c|}
\hline VARIABLE & DIMENSIONES & Indicador \\
\hline $\begin{array}{l}\text { APTITUD } \\
\text { FÍSICA }\end{array}$ & $\begin{array}{l}\text { - Capacidad aeróbica } \\
\text { - Resistencia y fuerza abdominal } \\
\text { - Fuerza y flexibilidad de los músculos } \\
\text { de la extremidad superior } \\
\text { - Elevaciones del tronco en extensión } \\
\text { - Fuerza explosiva }\end{array}$ & $\begin{array}{l}\text { - Carrera/ marcha de la milla (1600 metros) } \\
\text { - Abdominal } 30 \mathrm{~s} \\
\text { - Push-up (Flexiones de brazos - } 90^{\circ} \text { ) } \\
\text { - Trunk-lift } \\
\text { - Burpee ( } 30 \mathrm{~s})\end{array}$ \\
\hline
\end{tabular}




\section{Resultados}

Al describir las características de la composición corporal, como se observa en la tabla 2, se evaluó las categorías ponderales del IMC del sexo femenino, donde se encontró a 51 estudiantes (29.6\%), ubicados dentro del peso ideal, y 27 estudiantes (15.7\%) preobesos.

Tabla 2.- Categoría ponderal de los valores del IMC de los estudiantes del sexo femenino (porcentaje entre paréntesis)

\begin{tabular}{cccccccc}
\hline $\begin{array}{c}\text { CATEGORÍA } \\
\text { PONDERAL }\end{array}$ & $\begin{array}{c}\text { Demasiado } \\
\text { bajo }\end{array}$ & $\begin{array}{c}\text { Peso } \\
\text { ideal }\end{array}$ & Preobeso & Obeso & $\begin{array}{c}\text { Muy } \\
\text { Obeso }\end{array}$ & $\begin{array}{c}\text { Excesivamente } \\
\text { Obeso }\end{array}$ & TOTAL \\
\hline $\mathrm{n}^{\circ}$ & 89 & 51 & 27 & 4 & 1 & 0 & 172 \\
\hline$\%$ & $(51.7)$ & $(29.6)$ & $(15.7)$ & $(2.3)$ & $(0.6)$ & $(0.0)$ & $(100.0)$ \\
\hline
\end{tabular}

Asimismo, se observa en la tabla 3, al evaluar a los estudiantes del sexo masculino, 34 estudiantes (23.3\%) están ubicados dentro del peso ideal, y 23 estudiantes $(15.8 \%)$ son preobesos.

Tabla 3.- Categoria ponderal de los valores del IMC de los estudiantes del sexo masculino (porcentaje entre paréntesis)

\begin{tabular}{cccccccc}
\hline $\begin{array}{c}\text { CATEGORÍA } \\
\text { PONDERAL }\end{array}$ & $\begin{array}{c}\text { Demasiado } \\
\text { bajo }\end{array}$ & $\begin{array}{c}\text { Peso } \\
\text { ideal }\end{array}$ & Preobeso & Obeso & $\begin{array}{c}\text { Muy } \\
\text { Obeso }\end{array}$ & $\begin{array}{c}\text { Excesivamente } \\
\text { Obeso }\end{array}$ & TOTAL \\
\hline $\mathrm{n}^{\circ}$ & 85 & 34 & 23 & 4 & 0 & 0 & 146 \\
\hline$\%$ & $(58.2)$ & $(23.3)$ & $(15.8)$ & $(2.7)$ & $(0.0)$ & $(0.0)$ & $(0.0)$ \\
\hline
\end{tabular}

Es evidente que de la totalidad de los estudiantes de ambos sexos ( $n=318$ ), como se observa en la tabla 4, el $26.73 \%(85)$, se encuentran en el peso ideal y el $54.72 \%$ (174) se encuentran en el peso demasiado bajo. Asimismo, el $15.73 \%(50)$ del total de los estudiantes se ubican dentro de los preobesos.

Tabla 4.- Categoría ponderal del peso: demasiado bajo, peso ideal, preobeso, obeso, muy obeso y excesivamente obeso, del IMC de los estudiantes del 1 . $^{\text {er }}$ ciclo de estudios (porcentaje entre paréntesis).

\begin{tabular}{ccccccc}
\hline SEXO & $\begin{array}{c}\text { Demasiado } \\
\text { bajo }\end{array}$ & $\begin{array}{c}\text { Peso } \\
\text { ideal }\end{array}$ & Preobeso & Obeso & $\begin{array}{c}\text { Muy } \\
\text { Obeso }\end{array}$ & $\begin{array}{c}\text { Excesivamente } \\
\text { Obeso }\end{array}$ \\
\hline Femenino & $(51.15)$ & $(60.00)$ & $(54.00)$ & $(50.00)$ & $(100.00)$ & $(0.00)$ \\
Masculino & $(48.85)$ & $(40.00)$ & $(46.00)$ & $(50.00)$ & $(00.00)$ & $(0.00)$ \\
Total & $(100)$ & $(100)$ & $(100)$ & $(100.00)$ & $(100.00)$ & $(0.00)$ \\
$n$ & 174 & 85 & 50 & 8 & 1 & 0 \\
$\%$ & $(54.72)$ & $(26.73)$ & $(15.73)$ & $(2.51)$ & $(0.31)$ & $(0.00)$ \\
\hline
\end{tabular}


Del mismo modo, la tabla 5 muestra los valores medios, mínimos y máximos del IMC, donde los valores medios del IMC de las mujeres son mayores en casi todas las edades, excepto a los 19 años, donde los varones incrementan sus valores medios.

Tabla 5.- Media, valores mínimos y máximos del IMC en diferentes edades y ambos sexos

\begin{tabular}{lllllll}
\hline & \multicolumn{3}{c}{ FEMENINO } & \multicolumn{3}{l}{ MASCULINO } \\
\hline Edad & Media & Mínimo & Máximo & Media & Mínimo & Máximo \\
\hline 16 & 22.0 & 18.7 & 29.5 & 21.6 & 18.0 & 31.1 \\
17 & 22.3 & 17.8 & 29.6 & 22.2 & 16.0 & 32.4 \\
18 & 22.6 & 16.7 & 39.7 & 22.0 & 19.4 & 27.7 \\
19 & 22.5 & 18.7 & 28.3 & 23.0 & 17.8 & 29.8 \\
\hline
\end{tabular}

Por otro lado, en la tabla 6, se observa los valores medios, mínimos y máximos de la sumatoria de pliegues en ambos sexos y edades. Se observa que en el sexo masculino existe un incremento progresivo de porcentaje de grasa entre los 17 y 18 años en relación con las mujeres, pero que a los 19 años sus valores medios decrecen.

Tabla 6.- Media, valores mínimos y máximos de la sumatoria de pliegues en diferentes edades y ambos sexos

\begin{tabular}{lllllll}
\hline & \multicolumn{3}{c}{ FEMENINO } & \multicolumn{3}{c}{ MASCULINO } \\
\hline Edad & Media & Mínimo & Máximo & Media & Mínimo & Máximo \\
\hline 16 & 39.5 & 17.5 & 92.0 & 38.8 & 13.0 & 65.0 \\
17 & 35.7 & 16.5 & 67.0 & 38.5 & 16.5 & 75.0 \\
18 & 34.1 & 17.0 & 79.5 & 36.6 & 18.5 & 67.0 \\
19 & 38.5 & 22.0 & 63.5 & 36.9 & 16.5 & 65.0 \\
\hline
\end{tabular}

Posteriormente, se evaluó las características de las cinco pruebas de la aptitud física asociadas a la salud en función de la edad y sexo, donde se obtuvo los siguientes resultados: En la prueba abdominal (30 s), como muestra la tabla 7, se presentan los valores medios en ambos sexos y edades. Se comprobó que a la edad de 16, 18 y 19 años, los varones incrementaron sus valores medios en relación a las mujeres. 


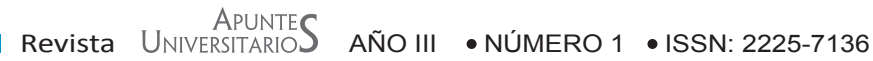

Tabla 7.- Media de la prueba abdominal de $30 \mathrm{~s}$ en diferentes edades de ambos sexos

\begin{tabular}{ccc}
\hline & FEMENINO & MASCULINO \\
\hline Edad & Media & Media \\
\hline 16 & 14 & 16 \\
17 & 15 & 13 \\
18 & 15 & 15 \\
19 & 16 & 16 \\
\hline
\end{tabular}

En la tabla 8 se muestra los valores medios de la prueba de Push Up de los estudiantes de ambos sexos y edades. Se observa que a la edad entre 17 y 19 años, los valores medios de los varones se encuentran por encima de los valores medios de las mujeres.

Tabla 8.- Media de la prueba Push Up en diferentes edades y ambos sexos

\begin{tabular}{ccc}
\hline & FEMENINO & MASCULINO \\
\hline Edad & Media & Media \\
\hline 16 & 9 & 8 \\
17 & 11 & 12 \\
18 & 13 & 12 \\
19 & 11 & 13 \\
\hline
\end{tabular}

En la tabla 9 se muestra los valores medios de la prueba Burpee (30 s) de los estudiantes de ambos sexos y edades. Se observa que, a los 16 años, las damas obtienen los valores medios por encima de los varones, pero que a la edad entre 17, 18 y 19 años, los varones incrementan sus valores medios.

Tabla 9.- Media de la prueba Burpee $30 \mathrm{~s}$ en diferentes edades y ambos sexos

\begin{tabular}{ccc}
\hline & FEMENINO & MASCULINO \\
\hline Edad & Media & Media \\
\hline 16 & 11 & 10 \\
17 & 11 & 10 \\
18 & 12 & 14 \\
19 & 10 & 11 \\
\hline
\end{tabular}


Al observar la tabla 10 de la prueba de Trunk Lift, se observa que a los 16 años las damas obtienen los valores medios por encima de los varones, pero que a la edad de 17, 18 y 19 años los varones incrementan sus valores medios.

Tabla 10.- Media de la prueba de Trunk Lift (cm) en diferentes edades y ambos sexos

\begin{tabular}{ccc}
\hline & FEMENINO & MASCULINO \\
\hline Edad & Media & Media \\
\hline 16 & 40.0 & 33.2 \\
17 & 32.4 & 37.9 \\
18 & 32.9 & 33.1 \\
19 & 33.0 & 33.8 \\
\hline
\end{tabular}

Finalmente, en la prueba de la Milla (Tabla 11), se comprobó que los valores medios decrecen en ambos sexos a lo largo de la edad, esto significa un mejor desempeño, dado que necesitan menos tiempo para cubrir la distancia considerada. Los varones presentan una ventaja clara entre las edades y 16 a 18 años, pero a la edad de 19 años, las mujeres tienen los valores medios superiores.

Tabla 11.- Media de la prueba Carrera de la Milla en diferentes edades y ambos sexos

\begin{tabular}{ccc}
\hline & FEMENINO & MASCULINO \\
\hline Edad & Media & Media \\
\hline 16 & 9.45 & 10.03 \\
17 & 9.12 & 9.80 \\
18 & 8.93 & 9.31 \\
19 & 9.92 & 8.56 \\
\hline
\end{tabular}

\section{Discusión}

Una de las constataciones que surgen del presente estudio en la evaluación de la aptitud física de los varones es que presentan valores medios superiores 
en casi todas las pruebas entre los 17, 18 y 19 años en relación a las damas, a excepción de los 16 años donde los valores medios de las damas supera a los de los varones en las pruebas de Push Up, Burpee 30 s y Trunk Lift.

Del punto de vista de la comparación entre ambos sexos, una de las constataciones que surgen de la aptitud física, en la evaluación de la Carrera de la Milla, es que los varones, en casi todas las edades, tienen mejores resultados. Estos resultados son, de algún modo, semejantes a los encontrados por Da Silva, Reis y Pires (2006).

Por otro lado, el comportamiento de los valores medios de las féminas en las pruebas de pliegues cutáneos es mayor que los varones en las edades entre 16 y 19 años, demostrando una similitud de los valores encontrados en los estudios de Berral (2001), Bustamante (2007) y Maia (2010)

La diferencia de los valores medios entre féminas y varones en la sumatoria de pliegues, sobre todo en la adolescencia (17 y 18 años), se debe a los cambios fisiológicos que se producen en estas edades. Esta información corrobora lo señalado por Malina (2002) que, durante la adolescencia, la masa grasa de las féminas aumenta casi dos veces más que la de los varones.

\section{Conclusiones}

Se concluye que el índice de masa corporal para ambos sexos, en sus valores medios, presenta un comportamiento ascendente semejante, y con ventajas mínimas a favor de las mujeres, excepto a los 19 años, donde los valores medios de los varones superan al de las damas.

En la sumatoria de los tres pliegues cutáneos analizados, los valores medios que presentan ambos sexos van disminuyendo conforme avanza la edad, esto es: 16,17 y 18 años y que, a los 19 años, sus valores medios aumentan.

En las pruebas de la aptitud física, en ambos sexos y a lo largo de la edad, existe un incremento significativo de los valores medios a favor de los varones, excepto la prueba de la Carrera de la Milla, donde a los 19 años las féminas obtienen mejores resultados; esto significa un menor desempeño, dado que necesitan menos tiempo para cubrir la distancia considerada.

\section{Recomendaciones}

Los resultados obtenidos reafirman la necesidad de proponer y planificar programas de actividad física constante y múltiple, donde se fomente la actividad para ambos sexos, con énfasis en las mujeres y donde los estudiantes 
los realicen con un mínimo de tres veces a la semana.

A su vez, la necesidad de implementar programas de educación alimentaria, estado nutricional y de prácticas de actividad física relacionados a la promoción de la salud. Que se conforme un equipo multidisciplinario para monitorear y hacer un seguimiento de los estudiantes que presentan sobrepeso y obesidad para derivarlos a los especialistas.

Que las entidades públicas relacionadas a la Educación Física y el deporte y las empresas privadas brinden su apoyo para la ejecución de estudios con una muestra representativa a nivel nacional y/o regional, y se logre el conocimiento de la población adolescente - juvenil peruana, puesto que los resultados serán una contribución a la Educación Física, la Cultura Física y el deporte.

Saulo Salinas

Universidad Peruana Unión email: saulo.salinas@upeu.edu.pe

Norma Luz Alejandría Lozano

Universidad Peruana Unión email: josemaria_200@hotmail.com

Recibido: 22 de marzo de 2013 Aceptado: 24 de mayo de 2013 


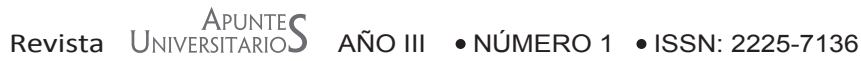

\section{Referencias}

Berral, F., Gómez, J., Viana, M., Berral, C., Benitez, C. (2001). Estudio de la Composición corporal en escolares de 10 a 14 años. Revista Brasileira de Cineantropometría y Desempenho Humano. 03 (1), $20-33$.

Bergmann, G., Braga, M., Garlipp, D., Lorenzi, T., Gaya, A. (2005). Alteración anual de crecimiento en la aptitud física relacionada a la salud de escolares. Revista Brasileira de Cineantropometría y Desempenho Humano. 07 (2), 55 - 61.

Bustamante, A., Seabra, A., Garganta, R., Maia, J. (2007). Efectos de la actividad física y del nivel socioeconómico en el sobrepeso y obesidad de escolares, Lima Este. Revista peruana de Medicina Experimental y Salud Pública. 24 (2), 1-11.

Da Silva, M. (2006). Crecimiento físico y aptitud física relacionada a la Salud de adolescentes de diferentes niveles socioeconómicos del colegio particular Luis Eduardo Magallanes - Brasil. Revista Brasileira de Cineantropometría y Desempenho Humano. 06 (2), 07 - 16.

Glaner, F. (2003). "Importancia de la aptitud física relacionada a la salud". Revista Brasileira de Cineantropometria y Desarrollo Humano. 5 (2), 75-85.

Heyward, V. y Stolarczyk, L. (2000). Evaluación de la composición corporal aplicada. 1. ${ }^{a}$ ed. Brasileira, 23, 73, 100, 101, 105.

Maia, J., Prista, A., Saranga, S., Nhantumbo, L., Silva, S. (2010). Padrón de adiposidad subcutánea en niños y jóvenes de Calanga: Puntas y vueltas de una simplicidad compleja, 59, 77.

Malina, R. y Bouchard, C. (2002). Actividad física del atleta joven. Sau Paulo,Brasil, 127

Pires, L., Ribeiro, J., Da Silva, R., Seabra, A., De Morais, F. (2004). Aptitud física. Revista Brasileira de Cineantropometría y Desempenho Humano. 06 (2), 07-16.

Reis, L. F. (2004). Estilo de vida, Antropometría y aptitud física relacionada a la Salud en escolares de Blumenau, SC. Revista brasileira de Cineantropometría y Desempenho Humano. $06(2), 88-2004$ 